\title{
Profiling the Lolium perenne Microbiome: From Seed to Seed
}

\author{
Ian Tannenbaum, ${ }^{1,2, \dagger}$ Jatinder Kaur, ${ }^{1}$ Ross Mann, ${ }^{1}$ Timothy Sawbridge, ${ }^{1,2}$ Brendan Rodoni, ${ }^{1,2}$ and German Spangenberg ${ }^{1,2}$ \\ ${ }^{1}$ Agriculture Victoria, AgriBio, Centre for AgriBioscience, Bundoora, Victoria, Australia \\ ${ }^{2}$ School of Applied Systems Biology, La Trobe University, Bundoora, Victoria, Australia
}

Accepted for publication 23 June 2020.

\section{ABSTRACT}

Lolium perenne (perennial ryegrass) is a common temperate pasture grass species and is favored by dairy farmers. Commercially, the known association with the mutualistic endophytic fungus Epichloë festucae subsp. lolii is used to enhance insect resistance of host plants. Knowledge of other members of the microbiome and their functions are limited. Sequencing of the V4 region of the $16 \mathrm{~S}$ ribosomal RNA gene was used to examine the bacterial microbiome of perennial ryegrass (Alto) seed (generation 1 [G1]), subsequent mature plants grown in soil and sand, and seed (generation 2 [G2]) from crosses of these mature plants. The G1 microbiome was dominated by the class Gammaproteobacteria. The mature plant microbiomes were far more diverse, comprising up to 37 classes inclusive of Gammaproteobacteria. Different growth media yielded different microbiome profiles in mature plants. The G2 microbiome, similar to the G1 microbiome, was dominated by Gammaproteobacteria as the primary constituent, with additional supplementation from class Bacilli. This suggests the continuation of a core microbiome which persists from seed through plant maturation to seed. This study sheds new light on the hereditability of perennial ryegrass bacterial microbiomes and has identified some operational taxonomic units of potential commercial significance due to their seed transmissibility, and their roles are currently being explored.

Keywords: 16S rRNA, bacterial recruitment, endophytes, Lolium perenne, metagenomics, microbial diversity, microbiome, microorganism, symbiosis, transmissibility
Plant microbiomes have piqued interest in agricultural research and have a range of commercial applications. Several microbes have been associated with certain beneficial functions within and around plants such as the well-established link between nitrogen fixation and Rhizobium spp. (Mabood et al. 2014). In return for providing nutrients, plants release exudates which provide a variety of metabolites that can be utilized by bacteria and fungi (Steinauer et al. 2016). Microorganisms have been utilized by their host for different uses such as nutrient acquisition and uptake, bioprotection, and bioremediation (Chaudhary and Khan 2018; de Souza et al. 2015; Wang et al. 2018).

Perennial ryegrass (Lolium perenne) is the most commonly used grazing grass in temperate Australian and New Zealand pasturelands, and symbiotic Epichlö̈ fungal endophytes are commercially exploited to provide insect resistance in some environments

${ }^{\dagger}$ Corresponding author: I. Tannenbaum; ian.tannenbaum@agriculture.vic.gov.au

Funding: Support was provided by DairyBio, Agriculture Victoria, Dairy Australia, and the Gardiner Dairy Foundation.

*The $\boldsymbol{e}$-Xtra logo stands for "electronic extra" and indicates there are supplementary materials published online.

The author(s) declare no conflict of interest.

(C) 2020 The American Phytopathological Society
(Ekanayake et al. 2012; Hettiarachchige et al. 2019; Kaur et al. 2015; Reed 2014). These Epichlö̈ endophytes are also seed transmissible, allowing their benefits to persist though seed generations. This can be viewed as a commercial product to microbially enhance perennial ryegrass seed and also an example of the holobiont concept. This conceptualizes plant functions being outsourced to members of the microbiome (Gilbert et al. 2012; Hassani et al. 2018). Resident fungi are only one part of the total holobiont, with other members including the bacterial epiphytes and endophytes, a far less studied collection of microorganisms (Khaksar et al. 2017).

Thus far, several plants have been evaluated to determine their microbiome structure. Arabidopsis thaliana, a model plant, was extensively characterized across root exudates, rhizosphere, phyllosphere, and transcriptome (Bai et al. 2015; Bulgarelli et al. 2012; Kerr et al. 2019; Lundberg et al. 2012; Strehmel et al. 2014). There has also been characterization of a variety of crop plants such as rice, maize, soybean, and wheat (Edwards et al. 2015; Gdanetz and Trail 2017; Hassani et al. in press; F. Liu et al. 2019; Peiffer and Ley 2013). Recent microbiome research around perennial ryegrass involved profiling root-associated bacteria exclusively or fungal endophytes, or was related to plant health and disease (Chen et al. 2016; Heineck et al. 2018; Ma et al. 2015; Nissinen et al. 2019).

All plants have multiple subcompartments comprising their microbiomes, the core components being the phyllosphere (aerial surfaces), endosphere (internal tissue and vasculature), and rhizosphere 
(root surface) (Shade et al. 2017; Turner et al. 2013). Microbial profiles have proven to be quite different between compartments (Edwards et al. 2015; Fernández-González et al. 2019). For instance, whereas the rhizosphere and root endosphere are in proximity, not all microorganisms have the mechanisms to enter the plant for colonization. This can yield different profiles between compartments with some shared constituents (Fernández-González et al. 2019). Frank et al. (2017) briefly discussed the modes of microbial transmission, horizontally from the environment and vertically from the parent plant.

During seed generation, a new compartment of the microbiome is created. Seed contain initial reservoirs of bacterial endophytes within the endosphere, inherited from their parent (Eyre et al. 2019; Raj et al. 2019). Some of these bacterial endophytes have been associated with germination, early plant health, and development (Felestrino et al. 2017; Huang et al. 2016). Such bacterial endophytes would be selected for during seed production and would be transmitted vertically, carried along with the vascular tissue to the seed heads. Once the seed microbiome assembly completes and becomes encapsulated within the seed coat, the microbial consortia become more difficult to influence until germination (Kim et al. 2020).

In this study, we described the bacterial microbiome of perennial ryegrass seed from two commercial batches of the Alto cultivar; one batch contained the commercially available fungal endophyte Epichlö festucae subsp. lolii and the other was an endophyte-free batch. Comparison of batches allowed us to infer the effect of resident endophytes on the host bacterial microbiome. An initial seedborne microbiome for perennial ryegrass was determined by assessing surface-sterilized seed; identified microbiota were presumed to originate from the seed. In addition, we monitored the microbiomes of our plants through their maturation and profiled the subsequent-generation seed to determine microbiome transmissibility across two different soil types (Walters et al. 2018). This study revealed a persistent microbiome of perennial ryegrass, from seed to seed, with accompaniments provided during plant maturation. The implications from these findings may assist in how pasture and crop health will be approached in the future, with the potential for reducing use of chemical additives and fertilizers.

\section{MATERIALS AND METHODS}

Ryegrass seed selection. Two seed batches of perennial ryegrass (L. perenne 'Alto') were sourced from Agriseeds New Zealand. Of the batches, one contained a standard endophyte (SE) (resident fungal endophyte $E$. festucae subsp. lolii) and one was without an endophyte (WE); these were referred to as Alto-SE and Alto-WE, respectively (Kaur et al. 2015). These batches were harvested in March 2015 and stored at $4^{\circ} \mathrm{C}$ until germination in November 2016. To determine SE presence or absence, a kompetitive allele-specific (KASP) assay (Supplementary Methods S1) was performed on each batch (Qureshi et al. 2018).

Sample preparation. Seed were surface sterilized as per Kaur et al. (2015), with the following modifications; the $\mathrm{H}_{2} \mathrm{SO}_{4}$ step was removed and seed was washed in $3 \% \mathrm{NaClO}$ for $3 \mathrm{~min}$, then washed five times in sterile Milli-Q $\mathrm{H}_{2} \mathrm{O}$ for $1 \mathrm{~min}$. Batches were germinated on moistened filter paper in sterile Petri dishes and incubated in the dark at room temperature for 7 days. Seedlings of similar size were harvested and sectioned into shoots and roots (Supplementary Fig. S3a), which represented the first-generation seed microbiome (G1). G1 seedlings from each batch were transplanted into two different soil types (a potting mix and an autoclaved mixture of sand and vermiculite, ratio 3:2) and incubated under glasshouse conditions for 10 weeks. Mature plants from each batch were harvested by removing three tillers with their respective roots, washed in sterile phosphate-buffered saline (PBS) (roots only), then sectioned into shoots and roots (Supplementary Fig. S3b), which represented the mature plant microbiome. The remaining plants from each batch were vernalized in a Conviron A1000 growth chamber (Conviron, Canada) with a cycle of $5^{\circ} \mathrm{C}$ for $16 \mathrm{~h}$ (night) and $14^{\circ} \mathrm{C}$ for $8 \mathrm{~h}$ (day) for 10 weeks. To induce flowering, the cycle was adjusted to $24^{\circ} \mathrm{C}$ for $20 \mathrm{~h}$ (day) and $16^{\circ} \mathrm{C}$ for $4 \mathrm{~h}$ (night), following which plants were allowed to cross-pollinate. The soil and sand/vermiculite pots remained separate during the vernalization process. SE and WE batches were allowed to vernalize together because SE is not known to be carried by pollen (J. Liu et al. 2017). The seed was harvested from plants of each batch, germinated as above, and sectioned into shoots and roots (Supplementary Fig. S3a), which represented the second-generation seed microbiome (G2). Full details regarding all treatment combinations are provided in Table 1. All plant sections were processed for DNA extraction and 16S amplicon sequencing.

DNA extraction and $16 \mathrm{~S}$ amplicon sequencing. For profiling the bacterial communities in seed (G1 and G2), 50 to 100 seedlings were selected from each batch. Parental soil type for G2 was recorded (Supplementary Table S1). Root and shoot sections were pooled into sets of 5, creating 10 replicates for each batch in G1 and 5 replicates of 5 pooled organs for G2 per parental plant. For profiling the bacterial communities in mature plants, shoots and roots were harvested from five plants per growth medium. DNA extraction was performed using the QIAGEN MagAttract 96 DNA Plant Core Kit according to the manufacturer's instructions, with minor modifications for use with a Biomek FX liquid handling station.

The bacterial microbiome was profiled targeting the V4 region of the $16 \mathrm{~S}$ ribosomal RNA (rRNA) gene according to the Illumina 16S Metagenomic Sequencing Library Preparation protocol (Supplementary Methods S2), with minor modification to include the use of peptide nucleic acid (PNA) PCR blockers to reduce amplification of 16S rRNA gene sequences derived from the plant organelle genome (Lundberg et al. 2013; Wagner et al. 2016). Paired-end sequencing was performed on the G1 and G2 libraries on an Illumina MiSeq using a $2 \times 300$ bp v3 chemistry and an Illumina HiSeq3000 using $2 \times 150$ bp, respectively. The mature plant bacterial libraries were prepared as previously stated and were sequenced on both aforementioned platforms using the same specifications. All Illumina sequences have been submitted to the NCBI Short Read Archive (SRA accession PRJNA577477).

Data processing and diversity analysis. Sequence data were trimmed and joined using PANDAseq (Massela et al. 2012). HiSeq3000 and MiSeq data were processed as described in the supporting information (Supplementary Method S2). Post PANDAseq reads were imported via manifest, as single read FASTQ sequences, into QIIME2 (release 2019.10) for further processing and organelle read removal (Bolyen et al. 2019). The q2-deblur plugin was used for dereplication and denoising (Amir et al. 2017). The q2-featureclassifier plugin was used for the taxonomic assignment of operational taxonomic units (OTUs) against the SILVA 1.32 database (released 12 December 2017). The q2-phylogeny plugin was used to generate rooted and unrooted phylogenetic trees by implementing MAFFT and FastTree 2 (Katoh and Standley 2013; Price et al. 2010). Comparative analysis of treatments was conducted using QIIME2 and Genedata Expressionist, Analyst Module (Genedata AG, Basel, Switzerland).

$\alpha$-Rarefaction plots were created using QIIME2 to optimize sample rarefaction to 5,000 reads per sample. The q2-diversity plugin was used to generate Jaccard dissimilarity plots between all treatments. Comparative analysis was conducted on the G1 Alto-SE and Alto-WE seed to profile the effects of fungal endophyte and 
organ on the bacterial microbiome. A brief analysis of PNA clamp use was conducted to ensure the prevention of organelle amplification in the 16S PCR and is available in the supporting information (Lundberg et al. 2013). Similar analyses were undertaken on the mature plants grown in sand and soil, as was G2. Genedata Expressionist, Analyst Module (Genedata AG, Basel, Switzerland) was used for the production of Venn diagrams to demonstrate commonalities between treatment and to infer transmissibility of microbial constituents.

Microbiome isolation. For G1 bacterial isolations, 50 seedlings were selected from Alto-WE and 25 seedlings were selected from Alto-SE. Each seedling was sectioned into shoots and roots (Supplementary Fig. S3a). Each section was pooled into a set of 5, creating 10 replicates for Alto-WE and 5 replicates for Alto-SE. Seedlings were ground two times in PBS in a Qiagen TissueLyser II for $1 \mathrm{~min}$ at $30 \mathrm{~Hz}$, inverted after each cycle. Macerates were serially diluted to create a $10^{-2}$-to- $10^{-4}$ series, plated onto Reasoner's 2A agar (R2A) (Oxoid, Australia), and incubated at room temperature for up to 4 weeks. Individual colonies were purified on R2A. Pure subcultures were further grown in Reasoner's 2A broth (Amyl, Australia) for 24 to $48 \mathrm{~h}$ and stored in $20 \%$ glycerol at $-80^{\circ} \mathrm{C}$. For fungal isolations, 15 seedlings were selected from Alto-WE and 15 seedlings were selected from Alto-SE. Seedlings were sectioned as above and plated directly onto half potato dextrose agar with tetracycline hydrochloride at $0.5 \% \mathrm{wt} \mathrm{vol}^{-1}$, yielding only E. festucae subsp. lolii from Alto-SE seed only (data not shown).

Identification of bacterial isolates was performed by sequencing the full-length 16S rRNA gene for 83 isolates. DNA was extracted from each isolate using a DNeasy Blood and Tissue kit (Qiagen) according to the manufacturer's instructions. Further details, including the primer sequence and PCR protocols, are available in the supporting information (Supplementary Methods S4). Forward and reverse sequences were examined for quality, assembled using
Sequencher (Gene Codes Corporation 2017), compared with the NCBI 16S rRNA (Bacteria and Archaea) database, and taxonomically assigned by the greatest sequence homology. The 16S rRNA sequences were assembled into a database for the mapping of Illumina V4 sequence data using NINJA-OPS (Al-Ghalith et al. 2016).

\section{RESULTS}

Bacterial microbiome profiling (class level). The most prominent class in both the G1 and G2 microbiomes was Gammaproteobacteria, accounting for 88.5 to $99.9 \%$ of the OTU abundance, with Alphaproteobacteria accounting for 0 to $2 \%$ (Fig. 1). The major difference between the G1 and G2 microbiomes was the presence of class Bacilli, which accounted for up to $10.9 \%$ in G2 and was absent in G1 (Fig. 1). For G1, shoots were dominated by Gammaproteobacteria (99.80 to $99.96 \%$ ), whereas roots contained Gammaproteobacteria (97.86 to $99.28 \%$ ), Alphaproteobacteria (0.70 to $2.00 \%$ ), and Actinobacteria (0.02 to $0.04 \%$ ) (Fig. 1). Bacteroidetes accounted for $0.10 \%$ of the OTU abundance in G1 shoots containing SE (Fig. 1). For G2, roots contained Gammaproteobacteria ( 96.42 to $99.89 \%$ ), Bacilli ( 0.07 to $1.48 \%$ ), and up to $2.02 \%$ of Alphaproteobacteria, whereas shoots contained less Gammaproteobacteria ( 88.5 to $98.36 \%$ ) and greater abundances of Bacilli (1.62 to $10.88 \%$ ) and Alphaproteobacteria (0.62 to $2.02 \%$ ) (Fig. 1). Presence of SE was associated with a greater abundance of Bacilli and Alphaproteobacteria in G2 shoots only (Fig. 1).

The mature plant microbiomes contained 18 to 37 classes (Fig. 1), predominantly comprising Gammaproteobacteria (18.03 to $31.64 \%$ ), Alphaproteobacteria (12.50 to $23.54 \%$ ), Bacteroidia (11.72 to $23.01 \%$ ), and Deltaproteobacteria (2.68 to $6.97 \%$ ). The remaining classes encompassed 6.28 to $18.91 \%$ of the OTU abundances (Fig. 1). Plants grown in soil had a greater association with Actinobacteria (19.38 to $30.82 \%$ ) when compared with

\begin{tabular}{|c|c|c|c|c|c|c|c|c|}
\hline \multicolumn{9}{|c|}{$\begin{array}{l}\text { TABLE } 1 \\
\text { Additional details describing the assessed treatments }\end{array}$} \\
\hline Sample name ${ }^{a}$ & Status $^{b}$ & Organ & Age (days) & Plant type & $G^{c}$ & Growth media & Samples per pool & Total pools \\
\hline Sand SE shoot & + & Shoot & 90 & Mature plant & 1 & Sand & 3 & 11 \\
\hline Sand WE root & - & Root & 90 & Mature plant & 1 & Sand & 3 & 10 \\
\hline G1 SE shoot & + & Shoot & 7 & Seedling & 1 & Filter paper & 5 & 8 \\
\hline G1 WE root & - & Root & 7 & Seedling & 1 & Filter paper & 5 & 10 \\
\hline G1 WE shoot & - & Shoot & 7 & Seedling & 1 & Filter paper & 5 & 10 \\
\hline G2 SE root & $+^{d}$ & Root & 7 & Seedling & 2 & Filter paper & 5 & 36 \\
\hline Soil SE root & + & Root & 90 & Mature plant & 1 & Soil & 3 & 10 \\
\hline Soil SE shoot & + & Shoot & 90 & Mature plant & 1 & Soil & 3 & 9 \\
\hline Soil WE root & - & Root & 90 & Mature plant & 1 & Soil & 3 & 7 \\
\hline Soil WE shoot & - & Shoot & 90 & Mature plant & 1 & Soil & 3 & 11 \\
\hline
\end{tabular}


plants grown in sand (1.93 to 3.20\%) (Fig. 1). Roots from soilborne plants contained additional classes when compared with shoots, with 12 extra classes observed with SE presence and 9 with SE absence (Fig. 1). Plants grown in sand had a greater association with Oxyphotobacteria (15.62 to $19.89 \%$ ) than plants grown in soil (0.92 to $0.15 \%$ ) (Fig. 1). Roots from sandborne plants contained seven additional classes when compared with shoots (Fig. 1). There were no observable differences in the bacterial microbiome of sandborne plants with respect to SE presence or absence (Fig. 1).

To assess the presence or absence of bacterial taxa between treatments, we conducted a qualitative Jaccard similarity principal component analysis using the q2-diversity module of QIIME2 (Fig. $2)$. When comparing all treatments, primary separation was observed between the seed (G1 and G2) and the mature plant (sand and soil) microbiomes along axis 1, accounting for $16.63 \%$ of the microbiome variation (Fig. 2A and B). Secondary separation was observed between the G1 and G2 microbiomes along axis 2, accounting for $10.35 \%$ of the microbiome variation (Fig. 2a). Tertiary separation was observed between the sand and soil mature plant microbiomes along axis 3, accounting for $7.043 \%$ of the microbiome variation (Fig. 2B). Quaternary separation was observed between the SE and WE treatments within G1 only (Fig. 2A). Quinary separation was observed between the shoots and roots of G1 only (Fig. 2A). There was an observable separation of G2 by the SE presence or absence status of the parental plant (Fig. 2A and D).

When comparing only the mature plant microbiomes, primary separation was observed between plants grown in sand and soil, accounting for $25.77 \%$ of the microbiome variation along axis 1 (Fig. 2C). Secondary separation was observed between the roots and shoots of both the sand and soil treatments, accounting for
$4.215 \%$ of the microbiome variation along axis 2 (Fig. 2C). Tertiary separation was observed between SE and WE treatments in the roots from plants grown in sand only (Fig. 2C). When comparing only the G1 and G2 microbiomes, primary separation was observed between the generation treatments, accounting for $19.26 \%$ of the microbiome variation along axis 1 . Secondary separation was observed between SE and WE treatments, accounting for $11.24 \%$ of the microbiome variation along axis 2 (Fig. 2D).

Bacterial microbiome profiling (genus level). The seed microbiome (G1 and G2) consisted of 115 unique genera, 8 of which were unique to $\mathrm{G} 1$ and 92 of which were unique to $\mathrm{G} 2$. Fifteen genera were vertically transmitted from G1 to G2 (Fig. 3). The microbiome of mature plants grown in soil consisted of 496 genera, 21 of which persisted from G1. The microbiome of mature plants grown in sand consisted of 342 genera, 19 of which persisted from $\mathrm{G} 1$. When considering only the mature plant microbiomes, 216 genera were unique to the soil treatment and 62 genera were unique to the sand treatment. In all, 19 and 20 OTUs persisted from G1 into the mature plants grown in sand and soil, respectively (Fig. 3 ), and 280 genera were shared between the sand and soil treatments (Fig. 3). The G2 microbiome shared 86 and 89 genera with the mature plants grown in soil and sand, respectively (Fig. 3). In total, 9 genera were shared between the G2 and mature plants grown in soil exclusively and 12 genera were shared between the G2 and the mature plants grown in sand exclusively (Fig. 3).

Bacterial microbiome isolations. In total, 284 bacteria were isolated from the G1 seed of Alto-SE $(n=131)$ and Alto-WE $(n=$ $153)$. The SE presence or absence status of each batch was confirmed before bacterial isolation (Supplementary Fig. S1). A greater number of bacteria were isolated from roots $(n=171)$ than shoots $(n=115)$.

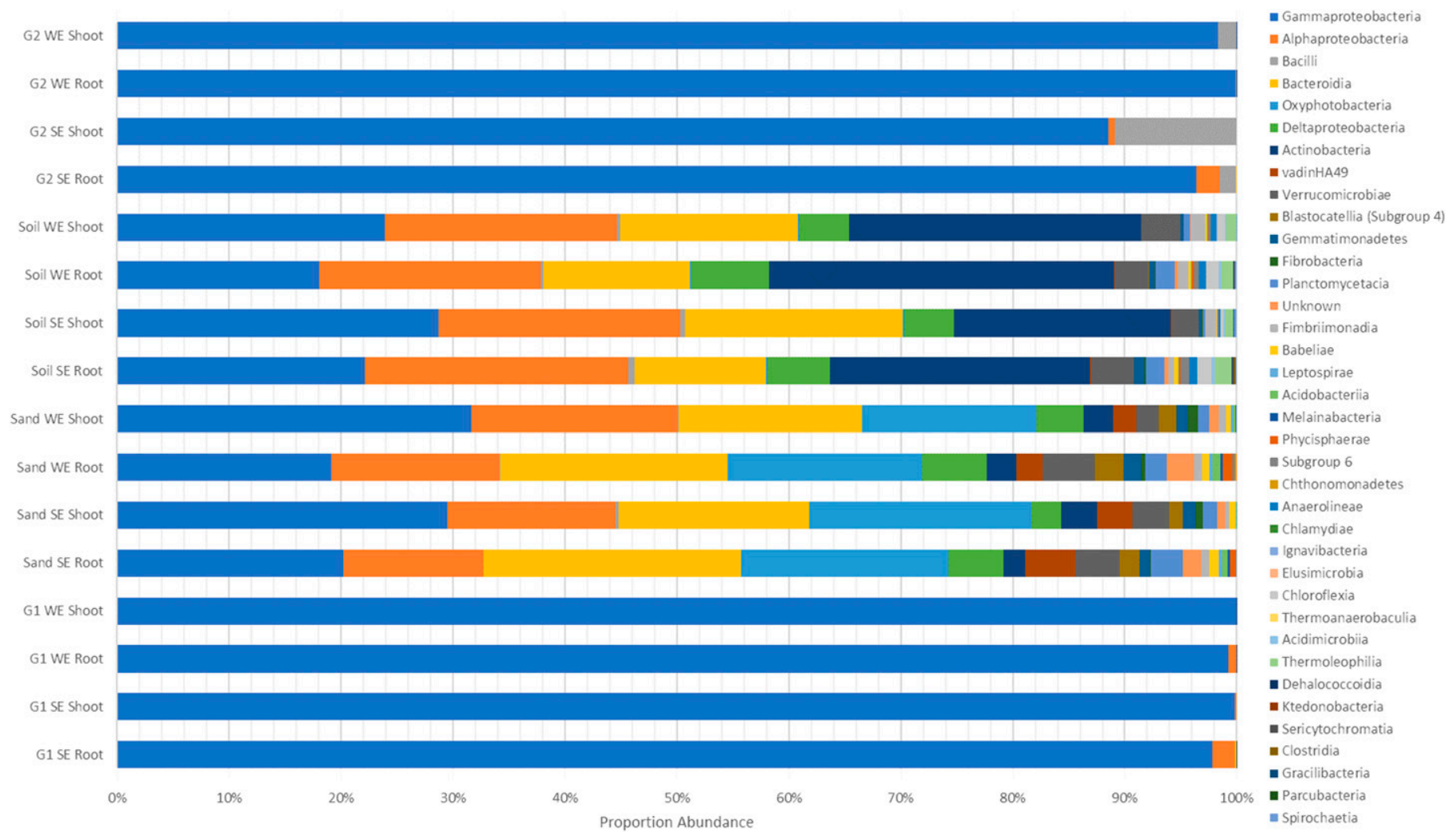

Fig. 1. Relative operational taxonomic unit abundance (class level) of the generation 1 (G1) mature plants grown in sand or soil and generation 2 (G2) Lolium perenne microbiomes by organ (root and shoot) and fungal endophyte presence (standard endophyte Epichloë festucae subsp. lolii [SE]) or absence (without endophyte [WE]). 
Sequencing of the $16 \mathrm{~S}$ ribosomal RNA gene. In all, 83 isolates were sequenced for the target 16S rRNA gene (Supplementary Method S4). There were determined to be 24 unique genera (Fig. 4). Alto-WE had greater diversity than SE at the genus level. Both seed batches had similar proportions of classes Gammaproteobacteria, Actinobacteria, Betaproteobacteria, and Bacilli. Isolates from family Bacteroidia were only observed in the absence of SE. Illumina V4 sequence data mapped to the full-length $16 \mathrm{~S}$ sequences from isolated bacteria (Supplementary Table S2). In total, 18 of the 24 culturable genera were detectable, where $93.6 \%$ of Illumina data mapped at $97 \%$ homology and $74.1 \%$ mapped at $99 \%$ homology (Supplementary Table S2). Further descriptions of the isolates are available in the supporting information (Supplementary Table S3).

\section{DISCUSSION}

The seed microbiome. The seed microbiome of perennial ryegrass (L. perenne) was dominated by Alphaproteobacteria and Gammaproteobacteria, similar to previous findings in a variety of alpine (Wassermann et al. 2019), Oryza spp. (Edwards et al. 2015),
Brassica spp., and other cropping species (Barret et al. 2015). Noticeably, the seed microbiome of genus Triticum, another member of the Poaceae family (Solanki et al. 2019), also included a pronounced Bacilli community. These higher classifications have been frequently observed in many other plant species' seed microbiomes, differing only in abundance between species (Turner et al. 2013). Shade et al. (2017) hypothesized that the seed microbiome reflects a key component of the previous parental plants' microbial consortia that may be used as a starting point for the establishment of a new microbiome assemblage in a daughter plant. Interestingly, the G2 microbiome was reflective of the G1 seed microbiome profile, with the addition of class Bacilli. The continuation of the prominent classes suggests the importance of the seedborne consortia in the viability of the seed for early development and germination (Torres-Cortés et al. 2018).

The mature plant microbiome. The mature plant microbiomes were far more complex than the seed microbiome. The proportion of Gammaproteobacteria was depleted in the mature plants and Alphaproteobacteria were enriched. Most of the OTUs detected in the mature plants were not detected in the G1 seed, suggesting that
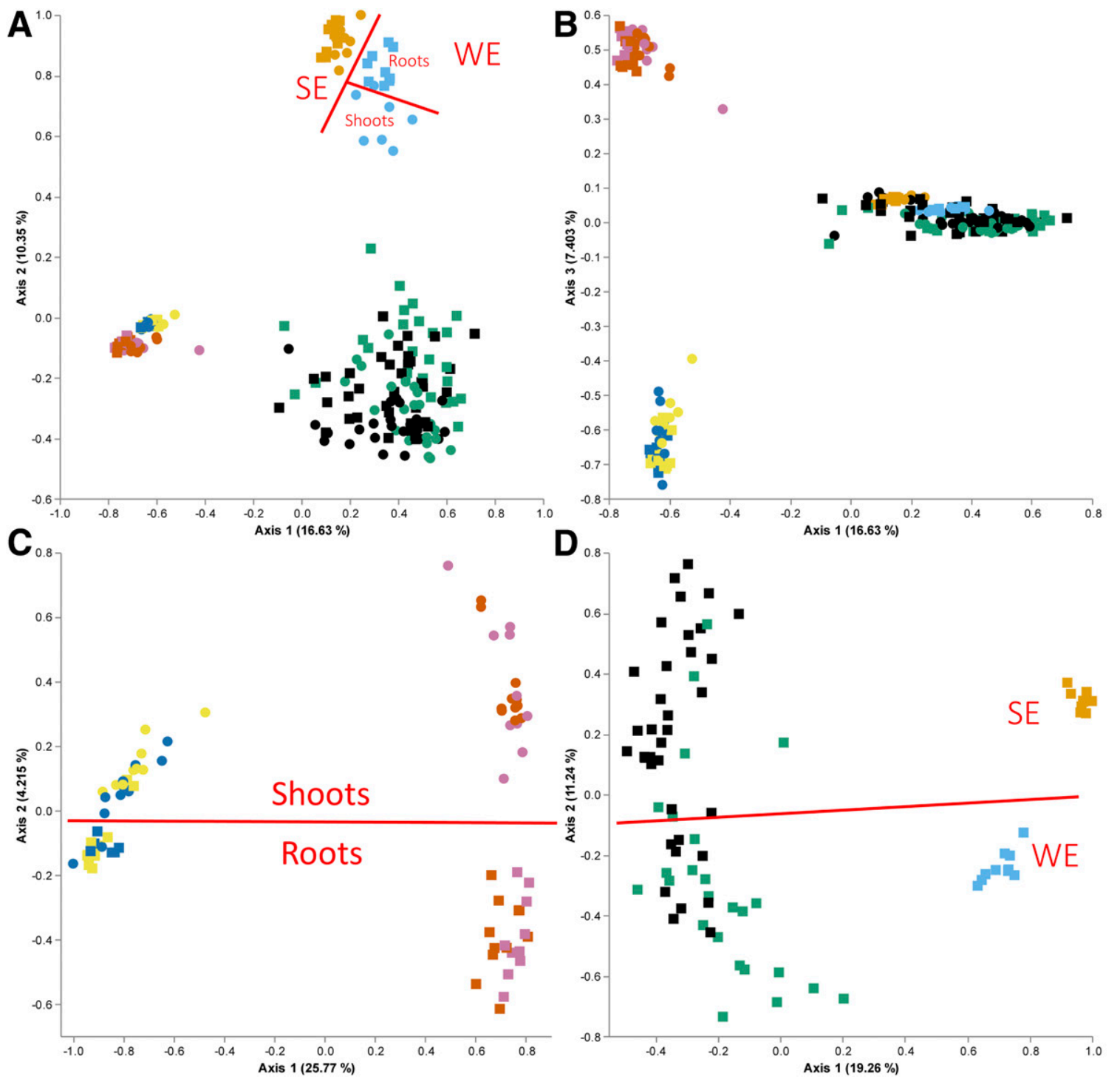

Sand SE Root

Sand SE Shoot

Sand WE Root

Sand WE Shoot

G2 SE Root

G2 SE Shoot

G2 WE Root

G2 WE Shoot

G1 SE Root

G1 SE Shoot

G1 WE Root

G1 WE Shoot

Soil SE Root

Soil SE Shoot

Soil WE Root

Soil WE Shoot

Fig. 2. Jaccard similarity plots describing the differences between the Lolium perenne bacterial microbiomes of seed (generation 1 [G1] and generation 2 [G2]) and mature plants (sand and soil). SE = standard endophyte Epichloë festucae subsp. Iolii and WE = without endophyte. A, All treatments (axis 1 and axis 2); B, all treatments (axis 1 and axis 3); C, mature plants (axis 1 and axis 2); and D, seed (axis 1 and axis 2) roots only. 
they were recruited from the environment and incorporated into the microbiome. In this study, the environment was observed to have the greatest effect on the microbiome of the mature plants. Similar

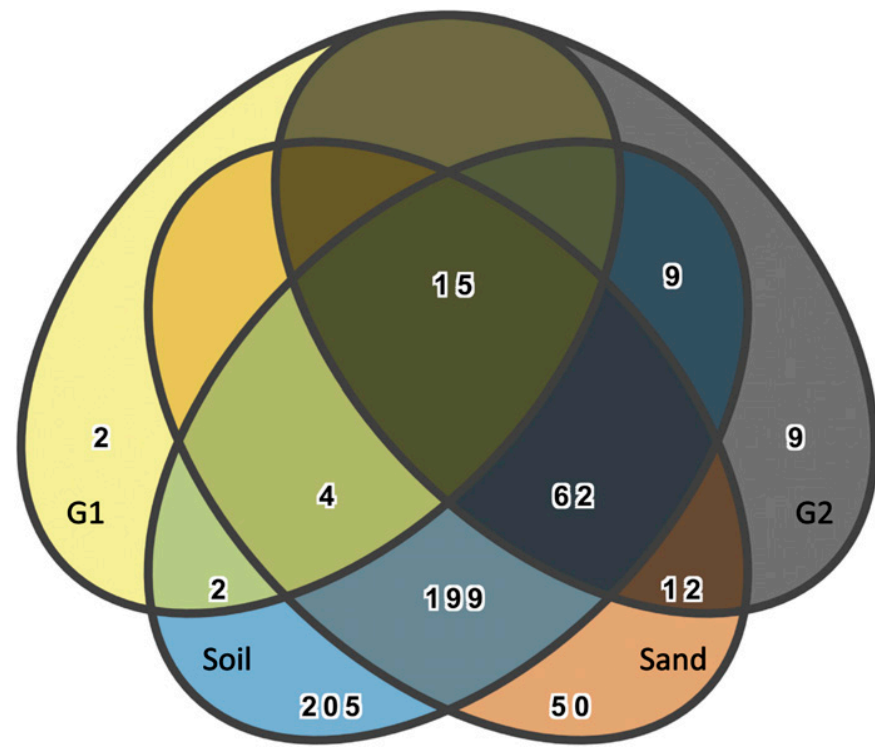

Fig. 3. Venn diagrams representing the number of identified genera between each of the assessed Lolium perenne microbiomes. G1 = generation 1 and G2 = generation 2 . observations were made by Schlatter et al. (2015) in their assessments of plant-soil community structures. When considering the differences between the sand and soil treatments, there were dramatic differences in the OTU populations in terms of the relative abundance reflecting the difference in media. This demonstrates that the plants likely actively recruited some OTUs which met their specific needs in each media type, as suggested by Gaiero et al. (2013) and Frank et al. (2017). From the loss of microbial richness in the progeny seed, we conclude that most of the microorganisms detected in the mature plants were transient in the evolution of the microbiome across the generation (Wani et al. 2015).

The intergenerational microbiome. This study has shown evidence of transmissible members of the bacterial microbiome between the parental plants and progeny seed. Similar to Epichloë fungal endophytes, some of the bacterial OTUs appeared to be conserved between generations. Most of the detected genera in this study from the G1 microbiome were conserved in the G2 microbiome. Many of the seedborne genera were depleted in the mature plants and were reenriched in the G2 microbiome. This suggests that a small proportion of the mature plant microbiome is particularly relevant for seed viability and establishment. Additionally, there was evidence of horizontal transmissibility from the environment, shown by OTUs observed in the G2 that were first detected in the mature plants. These observations imply that some bacteria taxa could be deployed in seed alongside Epichloë endophytes. The observed reduction of diversity between the mature plant and G2 microbiomes suggests that a strict set of selection criteria is applied by the mature plant during seed generation. If an

B

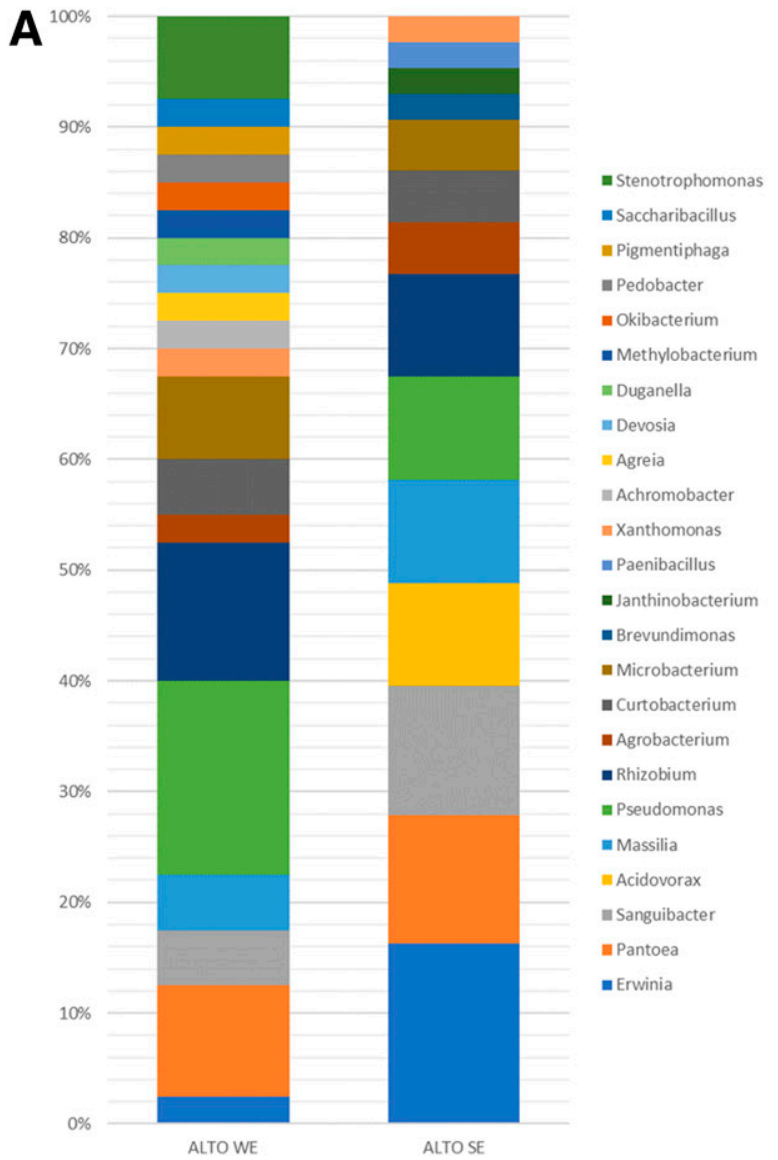

ALTO WE

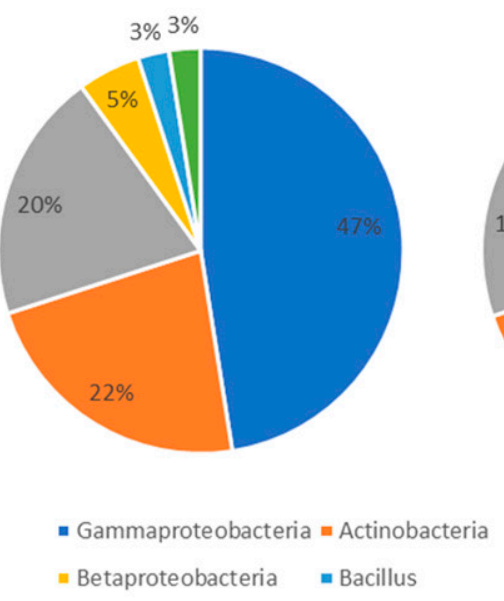

ALTO SE

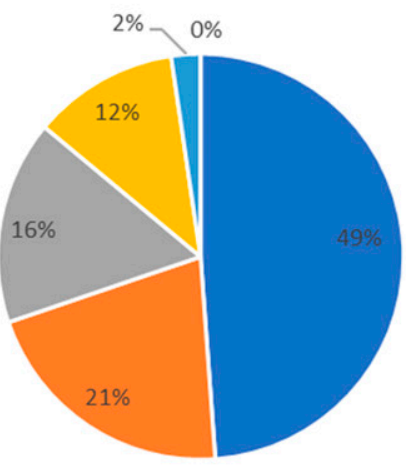

- Alphaproteobacteria

- Bacteroidia

Fig. 4. Proportion of genera as determined through A, 16 s ribosomal RNA sequencing of 83 isolates and $\mathbf{B}$, a summary at the class level. SE $=$ standard endophyte Epichloë festucae subsp. Iolii and WE = without endophyte. 
existing endophyte provides an indispensable function, the literature suggests that microorganisms will be more likely selected for during the reproductive stage and seed generation (Moran 2006).

The effect of organ and substrate on the microbiome. The major differences between the bacterial profiles of the mature plants were observed between the roots and shoots (Fig. 2C). These differences were driven by the presence or absence of some minor classes and the differential abundance of major classes. The roots were associated with greater microbial diversity, each being more reflective of the growth media. Because the sand used here was initially autoclaved, there was no preexisting microbiome within the growth medium. After 3 months of growth in a nonsterile greenhouse, the sterile sand likely became colonized by a wide array of microbia from multiple potential sources such as the seedling, water, airborne transmission from neighboring plants, and physical contact with neighboring plants. These results were similar to the demonstrated impacts of different soil types on the rhizosphere and endosphere populations of Populus spp. (Gottel et al. 2011). Because some rhizospheric bacteria possess mechanisms for plant colonization, they were capable of accessing and colonizing the endosphere (H. Liu et al. 2017). From our observations, the bacterial profiles of shoots were largely subsets of the roots (Edwards et al. 2015; Naylor et al. 2017). This suggests that some of the bacterial endophytes were transported from the roots, possibly by means of the plant vasculature and xylem vessels, to the shoots (Frank et al. 2017). A subset of the mature plant microbiome was observed in the $\mathrm{G} 2$ microbiome, suggesting that a bacterial subset was selected for during the reproductive phase and seed generation (Frank et al. 2017). Interestingly, the growth medium of the mature plants did not produce a clear separation in a Jaccard dissimilarity plot dependent on the parent plants' growth media, emphasizing the return on the seed microbiome (Supplementary Fig. S4b).

The effect of a resident fungal endophyte on the microbiome. There was an observed effect of the known seed-transmissible fungal endophyte E. festucae subsp. lolii on the G1 and G2 microbiomes within a cultivar; however, the difference were not observable in the mature plants (Figs. 1 and 2A and B) (Yang et al. 2013). One possible explanation for this could be related to the relative effect of a fungal endophyte, which primarily resides near the meristem, on the bacterial microbiome with respect to the host plant size. This could be further examined by finer sampling of the mature plant organs. The lack of endophyte effect was also observed by Nissinen et al. (2019) in their elucidation of fungal endophyte effects on the leaf microbiome of tall fescue. Although there is a clear, bulk effect in G1 and G2 (Fig. 1), the Jaccard plot shows more variation among the seed pools (Fig. 2A and D). Although the SE presence or absence status of G2 was unconfirmed by KASP, the differences seen in the Jaccard dissimilarity between SE and WE in G1 were along the same axis and direction as the G2 seedlings. If a portion of the microbiome can be transmitted via pollen, there may have been a mixture of the microbiomes during the vernalization process because the plants were matured together (Zasloff 2017). This implies that, within the Lolium genus, Epichloë endophyte status should be recorded when describing seed microbiomes.

Culturability of the microbiome. We obtained 284 isolates from the G1 microbiome from both shoots and roots of SE present and absent batches. The assessed subset of isolates aligned with 24 distinct genera. At class level, the isolations largely aligned to the 16S rRNA amplicon data, although Gammaproteobacteria were preferentially sequenced from plant tissue (Supplementary Table S2); more than 53 to 56 and 15 to $19 \%$ of the short reads mapped to isolates corresponding to the genera Pseudomonas and Erwinia, respectively. When also considering the low abundancy reads, we found that the culture-based approach largely represented the seed microbiome of G1 (Supplementary Table S2); 18 of the 24 genera were detectable at $97 \%$ homology using next-generation sequencing methods. Some close relatives of the culturable species have been described as plant growth promoting. Most notably, members of the genera Pseudomonas, Paenibacillus, Rhizobium, and Pantoea have been characterized with an array of beneficial effects, including nitrogen fixation, indole-3-acetic acid production, and inhibitory effects toward other bacteria and fungi (David et al. 2018; X. Liu et al. 2019; Patil et al. 2017; Silini-Cherif et al. 2012). Our isolates are currently being characterized to elucidate their contribution to the function of the L. perenne microbiome. The ability to culture members of a seed microbiome allows for screening processes to address the potential applications of bacterial endophytes and commercialization of those with beneficial effects (Sarhan et al. 2019).

\section{ACKNOWLEDGMENTS}

We thank D. Auer for his technical assistance.

\section{LITERATURE CITED}

Al-Ghalith, G. A., Montassier, E., Ward, D., and Knights, D. 2016. NINJA-OPS: Fast accurate marker gene alignment using concatenated ribosomes. PLOS Comput. Biol. 12:e1004658.

Amir, A., McDonald, D., Navas-Molina, J. A., Kopylova, E., Morton, J. T., Xu, Z. Z., Kightley, E. P., Thompson, L. R., Hyde, E. R., Gonzalez, A., and Knight, R. 2017. Deblur rapidly resolves single-nucleotide community sequence patterns. mSystems 2:e0191-16.

Bai, Y., Müller, D. B., Srinivas, G., Garrido-Oter, R., Potthoff, E., Rott, M., Dombrowski, N., Münch, P. C., Spaepen, S., Remus-Emsermann, M., Hüttel, B., McHardy, A. C., Vorholt, J. A., and Schulze-Lefert, P. 2015. Functional overlap of the Arabidopsis leaf and root microbiota. Nature 528: 364-369.

Barret, M., Briand, M., Bonneau, S., Preveaux, A., Valiere, S., Bouchez, O., Hunault, G., Simoneau, P., and Jacque, M.-A. 2015. Emergence shapes the structure of the seed microbiota. Appl. Environ. Microbiol. 81: 1257-1266.

Bolyen, E., Rideout, J. R., Dillon, M. R., Bokulich, N. A., Abnet, C. C., AlGhalith, G. A., et al. 2019. Reproducible, interactive, scalable and extensible microbiome data science using QIIME 2. Nat. Biotechnol. 37:852-857.

Bulgarelli, D., Rott, M., Schlaeppi, K., Ver Loren van Themaat, E., Ahmadinejad, N., Assenza, F., Rauf, P., Huettel, B., Reinhardt, R., Schmelzer, E., Peplies, J., Gloeckner, O., Amann, R., Eickhorst, T., and Schulze-Lefert, P. 2012. Revealing structure and assembly cues for Arabidopsis root-inhabiting bacterial microbiota. Nature 488:91-95.

Chaudhary, K., and Khan, S. 2018. Role of plant growth promoting bacteria (PGPB) for bioremediation of heavy metals. Pages 104-125 in: Biostimulation Remediation Technologies for Groundwater Contaminants. IGI Global, Hershey, PA.

Chen, L., Brookes, P. C., Xu, J., Zhang, J., Zhang, C., Zhou, X., and Luo, Y. 2016. Structural and functional differentiation of the root-associated bacterial microbiomes of perennial ryegrass. Soil Biol. Biochem. 98:1-10.

David, B. V., Chandrasehar, G., and Selvam, P. N. 2018. Pseudomonas fluorescens: A plant-growth-promoting rhizobacterium (PGPR) with potential role in biocontrol of pests of crops. Pages 221-243 in: Crop Improvement Through Microbial Biotechnology. Elsevier, Amsterdam, The Netherlands.

de Souza, R., Schoenfeld, R., and Passaglia, L. M. P. 2015. Bacterial inoculants for rice: Effects on nutrient uptake and growth promotion. Arch. Agron. Soil Sci. 62:561-569.

Edwards, J., Johnson, C., Santos-Medellin, C., Lurie, E., Podishetty, N. K., Bhatnagar, S., Eisen, J. A., and Sundaresan, V. 2015. Structure, variation, and assembly of the root-associated microbiomes of rice. Proc. Natl. Acad. Sci. U.S.A. 112:E911-E920.

Ekanayake, P. N., Hand, M. L., Spangenberg, G. C., Forster, J. W., and Guthridge, K. M. 2012. Genetic diversity and host specificity of fungal endophyte taxa in fescue pasture grasses. Crop Sci. 52:2243-2252. 
Eyre, A. W., Wang, M., Oh, Y., and Dean, R. A. 2019. Identification and characterization of the core rice seed microbiome. Phytobiomes J. 3:148-157.

Felestrino, E. B., Santiago, I. F., Freitas, L. D., Rosa, L. H., Ribeiro, S. P., and Moreira, L. M. 2017. Plant growth promoting bacteria associated with Langsdorffia hypogaea-rhizosphere-host biological interface: A neglected model of bacterial prospection. Front. Microbiol. 8:172.

Fernández-González, A. J., Villadas, P. J., Gómez-Lama Cabanás, C., ValverdeCorredor, A., Belaj, A., Mercado-Blanco, J., and Fernández-López, M. 2019. Defining the root endosphere and rhizosphere microbiomes from the World Olive Germplasm Collection. Sci. Rep. 9:20423.

Frank, A. C., Saldierna Guzman, J. P., and Shay, J. E. 2017. Transmission of bacterial endophytes. Microorganisms 5:70.

Gaiero, J. R., McCall, C. A., Thompson, K. A., Day, N. J., Best, A. S., and Dunfield, K. E. 2013. Inside the root microbiome: Bacterial root endophytes and plant growth promotion. Am. J. Bot. 100:1738-1750.

Gdanetz, K., and Trail, F. 2017. The wheat microbiome under four management strategies, and potential for endophytes in disease protection. Phytobiomes J. $1: 158-168$.

Gene Codes Corporation. 2017. Sequencher® version 5.4.6. DNA Sequence Analysis Software. Gene Codes Corporation, Ann Arbor, MI, U.S.A. http:// www.genecodes.com/

Gilbert, S. F., Sapp, J., and Tauber, A. I. 2012. A symbiotic view of life: We have never been individuals. Q. Rev. Biol. 87:325-341.

Gottel, N. R., Castro, H. F., Kerley, M., Yang, Z., Pelletier, D. A., Podar, M., Karpinets, T., Uberbacher, E., Tuskan, G. A., Vilgalys, R., Doktycz, M. J., and Schadt, C. W. 2011. Distinct microbial communities within the endosphere and rhizosphere of Populus deltoides roots across contrasting soil types. Appl. Environ. Microbiol. 77:5934-5944.

Hassani, M. A., Durán, P., and Hacquard, S. 2018. Microbial interactions within the plant holobiont. Microbiome 6:58.

Hassani, M. A., Özkurt, E., Franzenburg, S., and Stukenbrock, E. H. Ecological assembly processes of the bacterial and fungal microbiota of wild and domesticated wheat species. Phytobiomes J. In press. doi.org/10.1094/ PBIOMES-01-20-0001-SC

Heineck, G. C., Watkins, E., and Ehlke, N. J. 2018. The fungal endophyte Epichloë festucae var. lolii does not improve the freezing tolerance of perennial ryegrass. Crop Sci. 58:1788-1800.

Hettiarachchige, I. K., Elkins, A. C., Reddy, P., Mann, R. C., Guthridge, K. M., Sawbridge, T. I., Forster, J. W., and Spangenberg, G. C. 2019. Genetic modification of asexual Epichlö̈ endophytes with the perA gene for peramine biosynthesis. Mol. Genet. Genomics 294:315-328.

Huang, Y., Kuang, Z., Wang, W., and Cao, L. 2016. Exploring potential bacterial and fungal biocontrol agents transmitted from seeds to sprouts of wheat. Biol. Control 98:27-33.

Katoh, K., and Standley, D. M. 2013. MAFFT multiple sequence alignment software version 7: Improvements in performance and usability. Mol. Biol. Evol. 30:772-780.

Kaur, J., Ekanayake, P. N., Tian, P., van Zijll de Jong, E., Dobrowolski, M. P., Rochfort, S. J., Mann, R. C., Smith, K. F., Forster, J. W., Guthridge, K. M., and Spangenberg, G. C. 2015. Discovery and characterisation of novel asexual Epichloë endophytes from perennial ryegrass (Lolium perenne L.). Crop Pasture Sci. 66:1058.

Kerr, S. C., Gaiti, F., and Tanurdzic, M. 2019. De novo plant transcriptome assembly and annotation using Illumina RNA-Seq reads. Methods Mol. Biol. 1933:265-275

Khaksar, G., Treesubsuntorn, C., and Thiravetyan, P. 2017. Euphorbia miliinative bacteria interactions under airborne formaldehyde stress: Effect of epiphyte and endophyte inoculation in relation to IAA, ethylene and ROS levels. Plant Physiol. Biochem. 111:284-294.

Kim, H., Lee, K. K., Jeon, J., Harris, W. A., and Lee, Y.-H. 2020. Domestication of Oryza species eco-evolutionarily shapes bacterial and fungal communities in rice seed. Microbiome 8:20.

Liu, F., Hewezi, T., Lebeis, S. L., Pantalone, V., Grewal, P. S., and Staton, M. E. 2019. Soil indigenous microbiome and plant genotypes cooperatively modify soybean rhizosphere microbiome assembly. BMC Microbiol. 19:201.

Liu, H., Carvalhais, L. C., Crawford, M., Singh, E., Dennis, P. G., Pieterse, C. M. J., and Schenk, P. M. 2017. Inner plant values: Diversity, colonization and benefits from endophytic bacteria. Front. Microbiol. 8:2552.

Liu, J., Nagabhyru, P., and Schardl, C. L. 2017. Epichloë festucae endophytic growth in florets, seeds, and seedlings of perennial ryegrass (Lolium perenne). Mycologia 109:691-700.

Liu, X., Li, Q., Li, Y., Guan, G., and Chen, S. 2019. Paenibacillus strains with nitrogen fixation and multiple beneficial properties for promoting plant growth. PeerJ 7:e7445.
Lundberg, D. S., Lebeis, S. L., Paredes, S. H., Yourstone, S., Gehring, J., Malfatti, S., Tremblay, J., Engelbrektson, A., Kunin, V., del Rio, T. G., Edgar, R. C., Eickhorst, T., Ley, R. E., Hugenholtz, P., Tringe, S. G., and Dangl, J. L. 2012. Defining the core Arabidopsis thaliana root microbiome. Nature 488: 86-90.

Lundberg, D. S., Yourstone, S., Mieczkowski, P., Jones, C. D., and Dangl, J. L. 2013. Practical innovations for high-throughput amplicon sequencing. Nat. Methods 10:999-1002.

Ma, M., Christensen, M. J., and Nan, Z. 2015. Effects of the endophyte Epichlö̈ festucae var. lolii of perennial ryegrass (Lolium perenne) on indicators of oxidative stress from pathogenic fungi during seed germination and seedling growth. Eur. J. Plant Pathol. 141:571-583.

Mabood, F., Zhou, X., and Smith, D. L. 2014. Microbial signaling and plant growth promotion. Can. J. Plant Sci. 94:1051-1063.

Massela, A. P., Bartram, A. K., Truszkowski, J. M., Brown, D. G., and Neufeld, J. D. 2012. PANDAseq: PAired-eND Assembler for Illumina sequences. BMC Bioinf. 13:31.

Moran, N. A. 2006. Symbiosis. Curr. Biol. 16:R866-R871.

Naylor, D., DeGraaf, S., Purdom, E., and Coleman-Derr, D. 2017. Drought and host selection influence bacterial community dynamics in the grass root microbiome. ISME J. 11:2691-2704.

Nissinen, R., Helander, M., Kumar, M., and Saikkonen, K. 2019. Heritable Epichlö̈ symbiosis shapes fungal but not bacterial communities of plant leaves. Sci. Rep. 9:5253.

Patil, A., Kale, A., Ajane, G., Sheikh, R., and Patil, S. 2017. Plant growthpromoting rhizobium: Mechanisms and biotechnological prospective. Pages 105-134 in: Rhizobium Biology and Biotechnology. Soil Biology, vol. 50. A. P. Hansen, D. K. Choudhary, P. K. Agrawal, and A. Varma, eds. Springer, Cham, Switzerland.

Peiffer, J. A., and Ley, R. E. 2013. Exploring the maize rhizosphere microbiome in the field: A glimpse into a highly complex system. Commun. Integr. Biol. 6:e25177.

Price, M. N., Dehal, P. S., and Arkin, A. P. 2010. FastTree 2-Approximately maximum-likelihood trees for large alignments. PLoS One 5:e9490.

Qureshi, N., Bariana, H. S., Zhang, P., McIntosh, R., Bansal, U. K., Wong, D., Hayden, M. J., Dubcovsky, J., and Shankar, M. 2018. Genetic relationship of stripe rust resistance genes $Y r 34$ and $Y r 48$ in wheat and identification of linked KASP markers. Plant Dis. 102:413-420.

Raj, G., Shadab, M., Deka, S., Das, M., Baruah, J., Bharali, R., and Talukdar, N. C. 2019. Seed interior microbiome of rice genotypes indigenous to three agroecosystems of Indo-Burma biodiversity hotspot. BMC Genomics 20:924.

Reed, K. F. M. 2014. Perennial pasture grasses-An historical review of their introduction, use and development for southern Australia. Crop Pasture Sci. 65:691-712.

Sarhan, M. S., Hamza, M. A., Youssef, H. H., Patz, S., Becker, M., ElSawey, H., Nemr, R., Daanaa, H.-S. A., Mourad, E. F., Morsi, A. T., Abdelfadeel, M. R., Abbas, M. T., Fayez, M., Ruppel, S., and Hegazi, N. A. 2019. Culturomics of the plant prokaryotic microbiome and the dawn of plant-based culture media-A review. J. Adv. Res. 19:15-27.

Schlatter, D. C., Bakker, M. G., Bradeen, J. M., and Kinkel, L. L. 2015. Plant community richness and microbial interactions structure bacterial communities in soil. Ecology 96:134-142.

Shade, A., Jacques, M. A., and Barret, M. 2017. Ecological patterns of seed microbiome diversity, transmission, and assembly. Curr. Opin. Microbiol. 37: 15-22.

Silini-Cherif, H., Silini, A., Ghoul, M., and Yadav, S. 2012. Isolation and characterization of plant growth promoting traits of a rhizobacteria: Pantoea agglomerans lma2. Pak. J. Biol. Sci. 15:267-276.

Solanki, M. K., Abdelfattah, A., Britzi, M., Zakin, V., Wisniewski, M., Droby, S., and Sionov, E. 2019. Shifts in the composition of the microbiota of stored wheat grains in response to fumigation. Front. Microbiol. 10:1098.

Steinauer, K., Chatzinotas, A., and Eisenhauer, N. 2016. Root exudate cocktails: The link between plant diversity and soil microorganisms? Ecol. Evol. 6: 7387-7396.

Strehmel, N., Bottcher, C., Schmidt, S., and Scheel, D. 2014. Profiling of secondary metabolites in root exudates of Arabidopsis thaliana. Phytochemistry 108:35-46.

Torres-Cortés, G., Bonneau, S., Bouchez, O., Genthon, C., Briand, M., Jacques, M.-A., and Barret, M. 2018. Functional microbial features driving community assembly during seed germination and emergence. Front. Plant Sci. 9:902.

Turner, T. R., James, E. K., and Poole, P. S. 2013. The plant microbiome. Genome Biol. 14:209. 
Wagner, M. R., Lundberg, D. S., Del Rio, T. G., Tringe, S. G., Dangl, J. L., and Mitchell-Olds, T. 2016. Host genotype and age shape the leaf and root microbiomes of a wild perennial plant. Nat. Commun. 7:12151.

Walters, W. A., Jin, Z., Youngblut, N., Wallace, J. G., Sutter, J., Zhang, W., González-Peña, A., Peiffer, J., Koren, O., Shi, Q., Knight, R., del Rio, T. G., Tringe, S. G., Buckler, E. S., Dangl, J. L., and Ley, R. E. 2018. Large-scale replicated field study of maize rhizosphere identifies heritable microbes. Proc. Natl. Acad. Sci. U.S.A. 115:7368-7373.

Wang, X., Wang, C., Li, Q., Zhang, J., Ji, C., Sui, J., Liu, Z., Song, X., and Liu, $X$. 2018. Isolation and characterization of antagonistic bacteria with the potential for biocontrol of soil-borne wheat diseases. J. Appl. Microbiol. 125: 1868-1880.
Wani, Z. A., Ashraf, N., Mohiuddin, T., and Riyaz-Ul-Hassan, S. 2015. Plantendophyte symbiosis, an ecological perspective. Appl. Microbiol. Biotechnol. 99:2955-2965.

Wassermann, B., Cernava, T., Muller, H., Berg, C., and Berg, G. 2019. Seeds of native alpine plants host unique microbial communities embedded in crosskingdom networks. Microbiome 7:108.

Yang, T., Du, W., Zhou, J., Wang, X.-X., and Dai, C.-C. 2013. Effects of the symbiosis between fungal endophytes and Atractylodes lancea on rhizosphere and phyllosphere microbial communities. Symbiosis 61: 23-36.

Zasloff, M. 2017. Pollen has a microbiome: Implications for plant reproduction, insect pollination and human allergies. Environ. Microbiol. 19:1-2. 